\title{
Is something better than nothing?
}

\author{
Caroline Kowal, MD
}

$\mathrm{I}$ n July 2000 I spent 3 weeks in Haiti on a medical student elective. Eager to work in a new country and experience a new culture, I'd read about Haitian culture and history. This was my first exposure to medicine outside of North America. People warned me: "Haiti? Be careful! You can't trust anyone there! They're so poor, they'll take anything they can get their hands on!" I was confident common sense would keep me out of danger. I had a trusted supervisor and would be working in an excellent hospital. Haiti is a beautiful and mystical island. I wanted to experience the enchantment.

Some people persuaded me that Haitian hospitals would be short on supplies, so my supervisor and I collected medical supplies to donate. The hospital where I worked in Miami responded well to the request. They filled a box with supplies and marked it "Third World." It contained a motley collection of test tubes, syringes, gloves, diapers, alcohol swabs and old-fashioned Foley catheters. My supervisor (who'd been to Haiti several times) and I thought this would be a welcome and useful donation. "They'll love you at the hospital; they need everything they can get their hands on!" remarked my supervisor.

Shortly after I arrived in Haiti, a student helped me sort the mess of equipment. This proved arduous because it had been damaged and scattered during transit. But I still considered my efforts worthwhile; I'd been told, "something is always better than nothing."

When I delivered my donations and was shown the hospital's warehouse of medical supplies, I was surprised to see an abundance of new equipment - from laparoscopes to OR scrubs. I was embarrassed to learn that the equipment that I and many others had donated was not in short supply. This particular hospital was, in fact, experiencing a shortage of adhesive tape. Had I contacted them beforehand, I could have brought something useful rather than collecting random, outdated equipment they didn't need. For the price of shipping the donated supplies, I could have

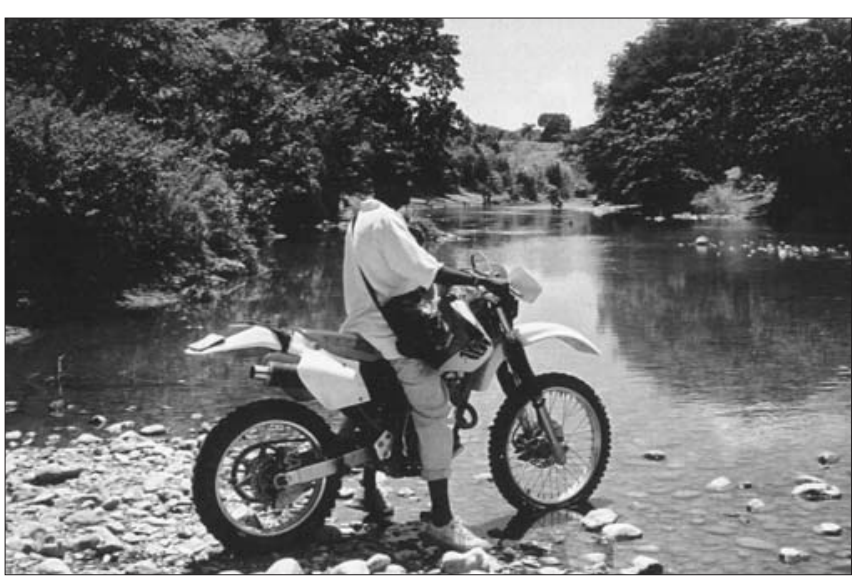

My ride to the countryside where mothers gather with their babies. We took immunizations in a small cooler. I prepared a speech in Creole about breastfeeding. We drove across the water to the path.

purchased a lot of adhesive tape.

The message from this experience is that sending just "anything" is not appropriate. The same standards used for selecting medical equipment for a hospital at home should also be applied to recipient hospitals in developing countries. Sending old equipment and expired medications is, at best, equivalent to giving nothing. Recipient hospitals often cannot use the donations because they do not serve their needs. And worse, unsolicited donations commonly cause additional problems for the recipient hospitals, creating storage problems or necessitating the disposal of medical garbage.

The Haitian hospital I worked at had received many other useless donations, like used blue pads (that looked clean but had been used by other patients) and a welding mask with the protective glass lenses missing. What use does a hospital have for a broken welding mask?

Another hospital I visited had received funds specifically donated for the construction of a new wing, even though their greatest need was a rebuilt sewage and electrical sys- 


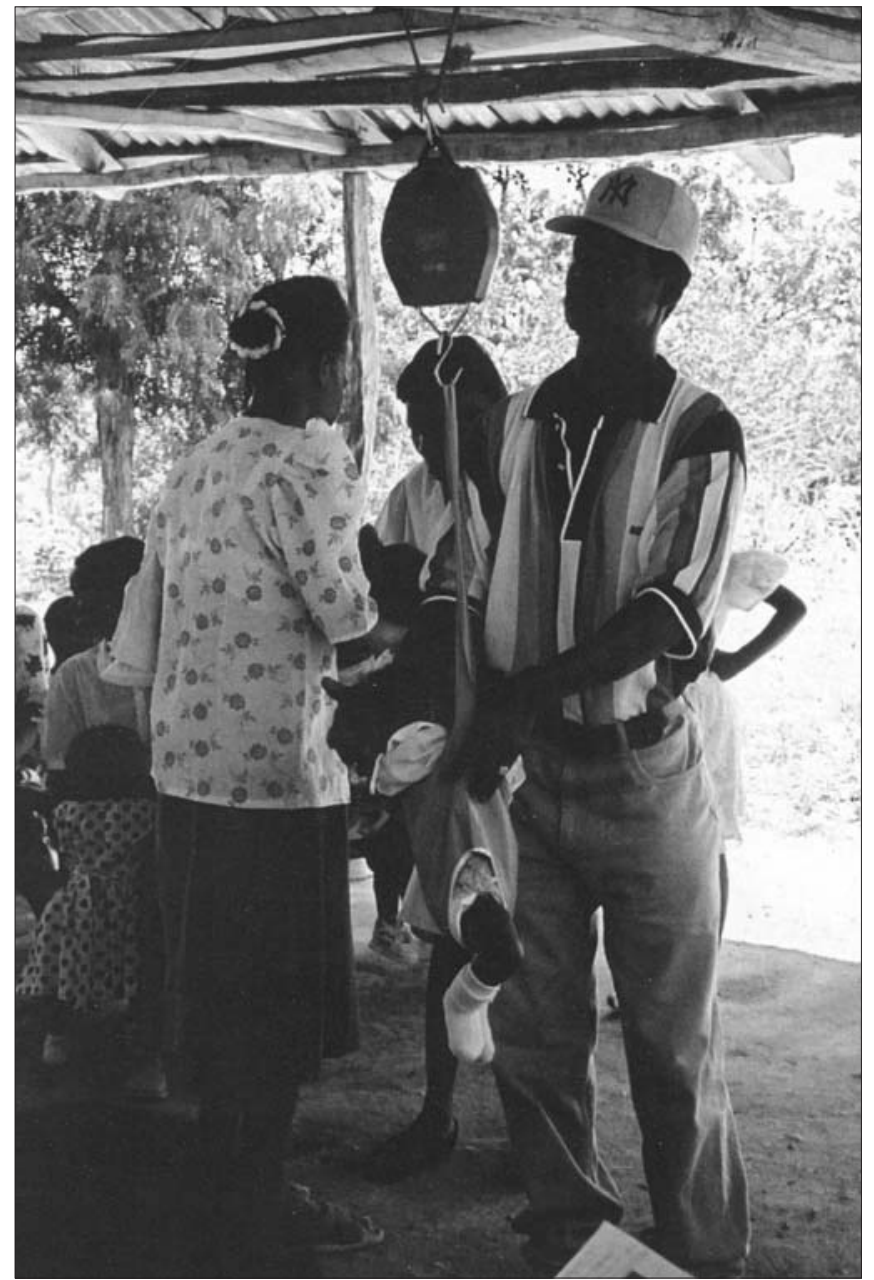

Weighing a baby at a public health gathering station. Mothers waited under a thatched roof.

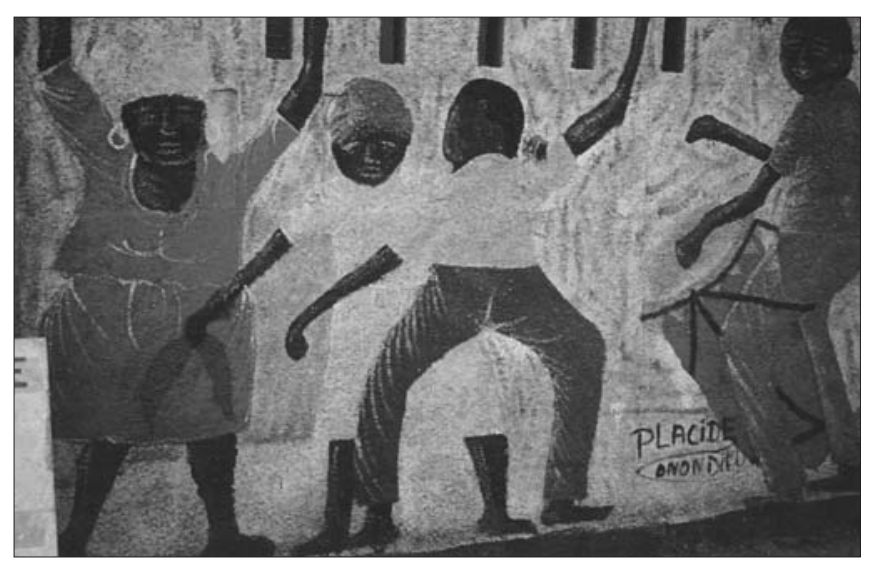

Wall mural along the waterfront of Cap Hatien in Northern Haiti.

tem. Had the donor not directed the funds, the hospital could have spent the money much more productively. Large donations may make the donor feel warm and fuzzy, and entitled to decide on the use of the money. Unfortu-

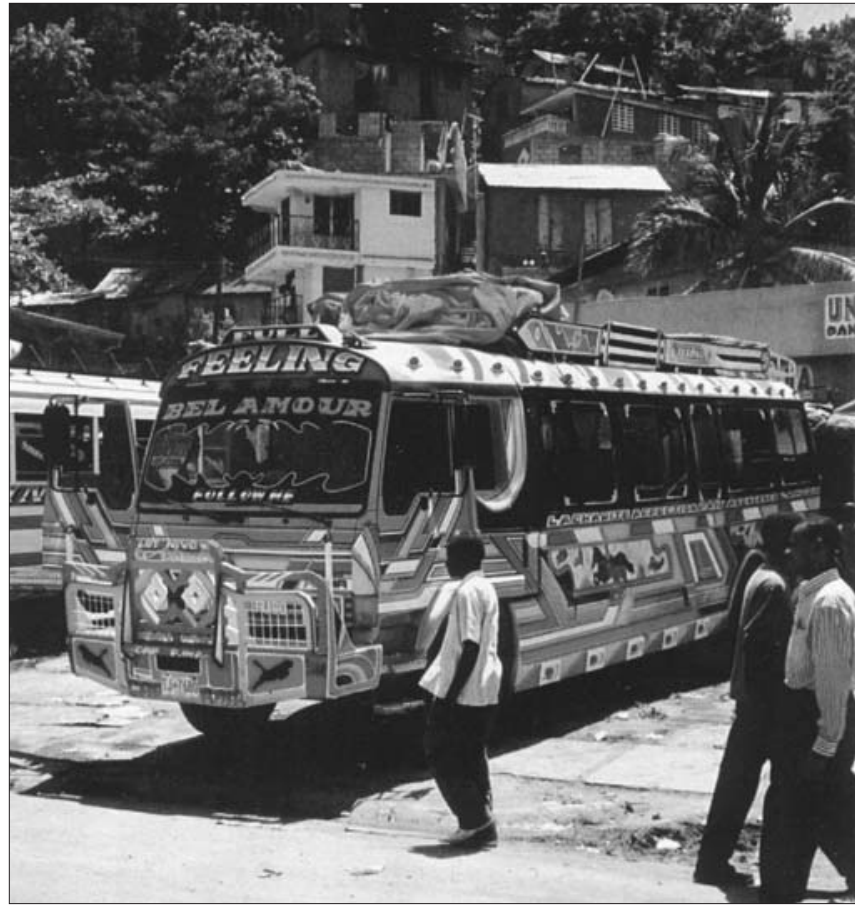

The party bus

nately, that doesn't always lead to the best use of desperately needed funds. Donors are not always aware of current needs.

Benefactors must change their approach to donating medical supplies. Medical professionals in developing countries are well aware of the latest medical technology and of new drug and treatment developments. Moreover, they are much more aware of what sort of donations or gifts will be most useful. Sending outdated or used supplies is a waste of time and money.

My advice for donated medical equipment and supplies is this.

1. Contact the hospital to find out what they need.

2. Sort and label all supplies and boxes prior to departure.

3. Send large quantities of the same item (e.g., a box of blue pads [unused!] instead of just one pad).

4. Rethink whether your efforts are for yourself or for those you wish to help.

And don't assume, as I did, that "something is better than nothing."

\section{Competing interests: None declared.}

Correspondence to: Dr. Caroline Kowal, Mount Sinai Hospital, 600 University Ave., Rm. 1465, Toronto ON M5G 1X5; fax 416 586-8885, carolinekowal@yahoo.com 